\title{
Coherent periodic activity in excitatory neural networks : the role of network connectivity
}

\author{
Alessandro Torcini ${ }^{1,2^{*}}$, Lorenzo Tattini ${ }^{1}$, Simona Olmi ${ }^{1,2}$ \\ From Twentieth Annual Computational Neuroscience Meeting: CNS*2011 \\ Stockholm, Sweden. 23-28 July 2011
}

Neural collective oscillations have been observed in very many context in brain circuits, ranging from ubiquitous

$\gamma$-oscillations to $\theta$-rythm in the hyppocampus. The origin of these oscillations is commonly associated to the balance between excitation and inhibition in the network, while purely excitatory circuits are believed to lead to "unstructured population bursts" [1]. However, coherent activity patterns have been observed also in "in vivo" measurements of the developing rodent neocortex and hyppocampus for a short period after birth, despite the fact that at this early stage the nature of the involved synapses is essentially excitatory [2]. Of particular interest are the so-called giant depolarizing potentials (GDPs), recurrent oscillations which repetitevely synchronizes a relatively small assembly of neurons and whose degree of synchrony is orchestrate by hub neurons [3]. Furthermore, in their recent study Bonifazi et al. [3] found that the functional connectivity of developing hippocampal networks is characterized by a power-law distribution of the output links with an exponent $\delta \sim 1.1-1.3$.

On the other hand, computational and theoretical studies of excitatory networks of leaky integrate-and-fire (LIF) neurons have revealed a regime characterized by coherent periodic activity at a macroscopic level both in fully [4] as well as in diluted networks [5]. This regime is characterized by a partial synchronization (PS) among the neurons reflected by a periodic behaviour of collective observables, while the single neuron evolution is quasiperiodic in time. The random dilution in the neuronal connectivity induces a weak form of chaos which vanishes for sufficiently large networks, therefore the PS regime appears to be robust with respect to this kind of disorder [6].

\footnotetext{
* Correspondence: alessandro.torcini@cnr.it

${ }^{1}$ CNR - Consiglio Nazionale delel Ricerche - Istituto dei Sistemi Complessi, 50019 Sesto Fiorentino, Italy

Full list of author information is available at the end of the article
}

In the same framework, namely pulse-coupled LIF excitatory networks, we have analyzed the role of the average degree of connectivity for the stability of the PS regime. In particular, we have considered directed random network with Erdös-Renyi topology with an average connectivity $\mathrm{C}$ corresponding to that of a finite scale-free network characterized by a power-law distribution with a decay exponent $1<\delta<2$. This amounts to have $C$ which scales as $\mathrm{N}^{\wedge}(2-\delta)$ with the number of neurons in the network. Therefore as two limiting cases massively connected networks are recovered for $\delta=1$ (where $\mathrm{C}$ is proportional to $\mathrm{N}$ ) and for $\delta=2$ sparse networks (where $\mathrm{C}$ is constant). Our analysis has revealed that, for a fixed system size $\mathrm{N}$, coherent periodic dynamics can be observed only for a connectivity $\mathrm{C}$ larger than a critical value $\Gamma=\Gamma(\mathrm{N})$ and that this value is independent from the exponent $\delta$. Furthermore, in the thermodynamic limit $\Gamma$ tends to an asymptotic value which depends only how the random connections are generated, namely statically or dynamically. The emergence of collective dynamics is controlled simply by the number of afferent synapses, independent of all the other details in the network topology, in agreement with what found for the synchronization of bursting neurons [6].

Finally, we have found indications that these systems are weakly chaotic, in the sense that in the thermodynamic limit completely regular dynamical behaviours are recovered [5]. Nonetheless, the degree of chaoticity increases by increasing $\delta$ and in the sparse network limit we expect to observe a constant maximal Lyapunov exponent, independent by $\mathrm{N}$.

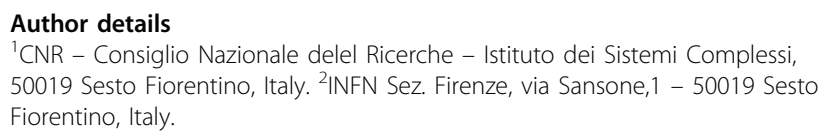

Published: 18 July 2011
C Biomed Central

C 2011 Torcini et al; licensee BioMed Central Ltd. This is an open access article distributed under the terms of the Creative Commons Attribution License (http://creativecommons.org/licenses/by/2.0), which permits unrestricted use, distribution, and reproduction in any medium, provided the original work is properly cited. 


\section{References}

1. Buszaki G: Rythms of the Brain. New York: Oxford University Press; 2006.

2. Allene C, Cattani A, Ackman JB, Bonifazi P, Aniksztejn L, Ben-Ari Y, Cossart R: Sequential Generation of Two Distinct Synapse-Driven Network Patterns in Developing Neocortex. J Neurosci 2008, 26:12851-12863.

3. Bonifazi P, Goldin M, Picardo MA, Jorquera I, Cattani A, Bianconi G, Represa A, Ben-Ari Y, Cossart R: GABAergic Hub Neurons Orchestrate Synchrony in Developing Hippocampal Networks. Science 2009, 326:1419-1424.

4. van Vreeswijk C: Partial synchronization in populations of pulse-coupled oscillators,. Phys. Rev. E 1996, 54:5522-5537.

5. Olmi S, Livi R, Politi A, Torcini A: Collective oscillations in disordered neural network.. Phys. Rev. E 2010, 81:046119.

6. Belykh I, de Lange E, Hasler M: Synchronization of Bursting Neurons: What Matters in the Network Topology. Phys. Rev. Lett 2005, 94:188101.

doi:10.1186/1471-2202-12-S1-P242

Cite this article as: Torcini et al: Coherent periodic activity in excitatory neural networks : the role of network connectivity. BMC Neuroscience 2011 12(Suppl 1):P242.

\section{Submit your next manuscript to BioMed Central} and take full advantage of:

- Convenient online submission

- Thorough peer review

- No space constraints or color figure charges

- Immediate publication on acceptance

- Inclusion in PubMed, CAS, Scopus and Google Scholar

- Research which is freely available for redistribution

Submit your manuscript at www.biomedcentral.com/submit 